\title{
Repurposing of chloroquine and hydroxychloroquine for the management of COVID-19
}

\author{
Fidelis A. Gberindyer ${ }^{1 *}$, Felix K. Shima ${ }^{2}$, Joel A. Bosha ${ }^{1}$, \\ Victor M. Ahur ${ }^{3}$, Festus T. Swem ${ }^{3}$, Matthew O. Abatan ${ }^{4}$
}

\begin{abstract}
${ }^{1}$ Department of Veterinary Pharmacology and Toxicology, ${ }^{3}$ Department of Veterinary Physiology and Biochemistry Federal University of Agriculture; PMB 2373, Makurdi, Nigeria

${ }^{2}$ Department of Veterinary Medicine, ${ }^{4}$ Department of Veterinary Pharmacology and Toxicology, University of Ibadan, Nigeria
\end{abstract}

Received: 25 July 2020

Revised: 01 October 2020

Accepted: 02 October 2020

*Correspondence:

Dr. Fidelis A. Gberindyer,

Email: fidegber@yahoo.com

Copyright: (c) the author(s), publisher and licensee Medip Academy. This is an open-access article distributed under the terms of the Creative Commons Attribution Non-Commercial License, which permits unrestricted non-commercial use, distribution, and reproduction in any medium, provided the original work is properly cited.

\begin{abstract}
The Coronavirus Disease-19 (COVID-19) pandemic has impacted adversely on the global health and socio-economic activities. There is currently no evidence-based anti-SARS-CoV-2 drug for COVID-19 therapy. This review highlights some pharmacological properties of chloroquine and hydroxychloroquine and prospects of repurposing them for the treatment of COVID-19. Google scholar was employed in searching relevant published journal articles $(n=118)$ in English. The search was later narrowed down to SARS-CoV-2, pathophysiology of COVID-19, available drugs for the management of COVID-19, clinical trials on repurposing drugs for COVID-19 therapy, and the role of chloroquine and hydroxychloroquine in the treatment of COVID-19. Documented evidence revealed that chloroquine and hydroxychloroquine have antiviral and immune-modulatory properties. Their antiviral effect is due to inhibition of the spike proteins of SARS-CoV-2 from binding to the cellular transmembrane receptors, angiotensin converting enzyme2 thereby preventing viral infections. Also, sequestration of these drugs into the lysosomes elevates lysosomal $\mathrm{pH}$ thus inhibiting lysosomal enzymatic functions vital for viral replication in those cells. Whereas, their immune-modulatory activity averts the inflammatory complications of COVID-19, particularly acute respiratory syndrome, by preventing cytokine storm through suppression of the production and putative release of pro-inflammatory cytokines. The adverse effects from these drugs, notably irreversible retinopathy and cardiac arrhythmia are rare but become life-threatening when they occur. These are minimal with hydroxychloroquine compared to chloroquine. Chloroquine and hydroxychloroquine could be repurposed for managing COVID-19 cases because they are already extensively used for treating acute nonresistant malaria and auto-immune diseases. Also, a viable vaccine cannot be available in the near future while there is a pressing need for treatments to lower the daily rise in morbidity and mortality associated with the disease. Nevertheless, we suggest that emphasis should be on hydroxychloroquine because of its superior antiviral effect and clinical safety.
\end{abstract}

Keywords: Angiotensin converting enzyme, 2, 4-aminoquinolines, Pandemic, SARS-CoV-2, Cytokine storm

\section{INTRODUCTION}

An outbreak of acute respiratory illness (pneumonia) of unknown etiology emerged in December 2019, in three patients from the city of Wuhan, Hubei Province, China. Subsequently, in January 2020, a novel coronavirus, later named "Severe Acute Respiratory SyndromeCoronavirus-2" (SARS-CoV-2) was identified as the cause of this disease; now referred to as "Coronavirus Disease2019" (COVID-19). At present, the spread of COVID-19 is out of proportion and is officially declared by the World Health Organization (WHO) as a pandemic since more 
than 100 countries have been affected with very high and increasing morbidity and mortality rates within a few months. ${ }^{1}$ This pandemic has not only impacted adversely on the global health, but also on socio-economic activities.

At present, there is no clinically validated and officially approved or recommended anti-SARS-CoV-2 drug. Supportive management protocol such as fluid and electrolytes therapy, oxygen supplementation, improved ventilation, and broad-spectrum antibacterial therapy against inevitable secondary bacterial infections is fundamental in managing COVID-19. ${ }^{2}$ During disease outbreaks of either epidemic or pandemic proportion, including COVID-19, time is usually a limitation to embark on new drug discovery and development; consequently physicians often try the existing drugs or drugs already into clinical trial but against a different condition peradventure they could effectively give the desired therapeutic outcome aside their originally validated and approved or intended clinical use, a practice referred to as drug "repurposing" or "repositioning". The aims of managing any epidemic or pandemic infectious disease are to successfully treat patients with confirmed cases, devise measures to circumvent or curtail the spread of the disease, and to protect the non-infected persons using vaccines. Since treatment of those infected is one of the key strategies for limiting the spread of infectious diseases, several treatment protocols have been proposed or adopted as off-label or on compassionate ground for the management and/or treatment of COVID-19 globally. One of these drugs is the use of chloroquine (CQ) or its analogue, hydroxychloroquine (HCQ) both with proven clinical efficacy against non-resistant malaria parasites and auto-immune diseases, including rheumatoid arthritis. ${ }^{3}$

The available reports on the use of CQ or HCQ for the treatment of COVID-19 has not been officially recommended/approved for now because such clinical trials had no control for comparison, and/or the sample size of the patients were too small, hence it would be irrational and premature to correlate the reported therapeutic outcomes (success or failures) with these drugs based on these studies. ${ }^{4,5}$ In addition, the in vitro inhibition of the SARS-CoV-2 by HCQ and the recommended dose regimen may not be translated into clinical efficacy until a controlled clinical trial is conducted on a required population of patients. ${ }^{6,7}$ Therefore, it is not plausible at the moment to establish the therapeutic effectiveness or otherwise of CQ and HCQ against SARS-CoV-2 infected patients as well as their clinical safety in patients based on anecdotal evidence. However, there are several ongoing well designed, controlled, randomized, and open-label clinical trials on the clinical efficacy and safety of CQ and HCQ in COVID-19 patients.

Because of the propensity of the development of resistance by pathogenic organisms, including viruses, the practice of drug repurposing ought to be handled with caution, particularly in pathogens whose characteristics are not fully understood, including SARS-CoV-2. This would probably circumvent the unintended consequences following the use of such drugs. It has been established that 4-aminoquinoline compounds, principally CQ and HCQ could cause serious adverse effects in patients such as irreversible retinal damage and cardiomyopathy even though the incidence is rare. ${ }^{9}$ Reports from unpublished sources, including the media have revealed that many people who are tested positive but asymptomatic, or those that are sure that they had contact with those tested positive who are either manifesting typical symptoms or not, or those that are not yet exposed have resorted to selfmedication with either CQ or HCQ for the inhibition, or for prophylactic purpose.

\section{OBJECTIVE}

Considering the inherent adverse effects of the 4aminoquinolines and the probability of the emergence of resistant strains of infectious agents following irrational self-medications, this concise review presents the therapeutic potentials and possible mechanisms of action of HCQ/CQ in ameliorating SARS-CoV-2 infection, as well as the adverse effects following the use of these drugs in patients.

\section{EVIDENCE ACQUISITION}

An extensive and synchronized review of original or review journal articles were executed between March and April, 2020. Google scholar search was conducted to obtain literatures from relevant English-published journals $(n=118)$ exclusively. We also employed secondary and hand searches of references list as well as other studies cross-indexed by the respective authors. Some of the major items searched included Coronaviruses and diseases caused by them including, SARS-CoV-2 and COVID-19, respectively; pathophysiology of COVID-19; clinical trials of drugs and vaccines for diseases caused by Coronaviruses, including COVID-19; therapeutic management of COVID-19; drug repurposing in diseases caused by coronaviruses, including COVID-19; and physicochemical and pharmacological properties of $\mathrm{CQ}$ and HCQ.

Even though the search was initially broad, it was subsequently narrowed down to the structure of SARSCoV-2; pathophysiology of COVID-19; available drugs for the management of COVID-19; concluded and ongoing clinical trials on repurposing drugs for COVID19 therapy; and the role of CQ and HCQ in the treatment of COVID-19. The authenticity of the information contained in the respective articles obtained through the search was evaluated based on the impact factor of the journal, the methodology employed, and the references given by the authors.

\section{DEVELOPMENT}

\section{Mechanism of action}

Chloroquine and its analogues, HCQ are alkylated 4aminoquinolines (Figure 1) which are weak bases. ${ }^{9}$ Hydroxychloroquine is more polar and less lipophilic 
compared to CQ, consequently with a lower rate and extent of absorption and volume of distribution. Chloroquine and its hydroxyl analogue, HCQ are racemic mixtures with equal amounts of $\mathrm{R}(-)$ and $\mathrm{S}(+)$ enantiomers which are traditionally used for the treatment of malaria, rheumatoid arthritis, and systemic lupus erythematosus. ${ }^{10}$ Both CQ and HCQ are potential drugs for the treatment of emerging viral infections due to their antiviral and immunomodulatory properties. ${ }^{11}$ Depending on the therapeutic target, potential anti-coronavirus therapies could be classified into those acting on the virus itself, and/or on the immune system or cells of the host. ${ }^{12}$

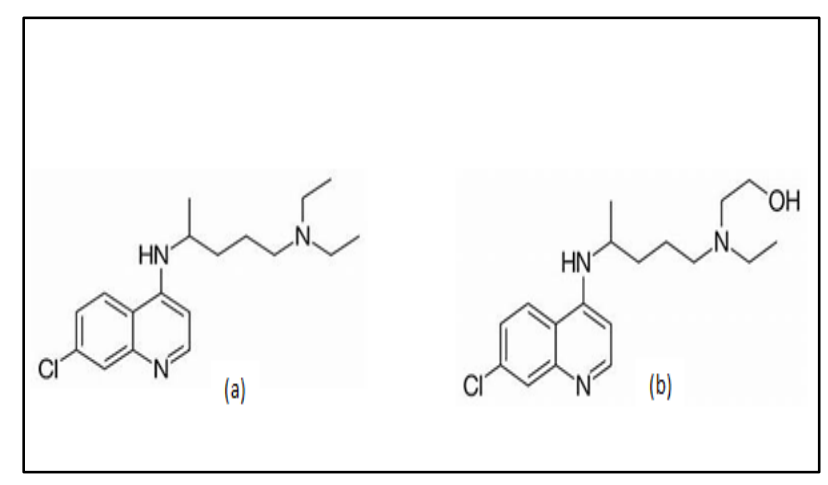

Figure 1: Chemical structures of chloroquine (a) and hydroxychloroquine (b).

\section{Antiviral effects}

Both CQ and HCQ exert direct antiviral effects as well as inhibit the $\mathrm{pH}$-dependent steps in the replication of several viruses, including members of the coronaviruses. ${ }^{11}$ Direct antiviral effects of CQ and HCQ are due to their inhibition of the endosome-mediated viral entry by blocking the virus from binding to transmembrane receptors of the cells, and inhibition of virus replication by acting on its critical enzymes. It has been reported that SARS-CoV-2 has four structural proteins, namely spike protein, envelope protein, membrane protein, and nucleocapsid protein. ${ }^{13,14}$

Spike proteins (SP) are the main proteins that interact and bind with the host cells receptor to mediate virus invasion of the target tissue or organ, consequently determining viral tissue or host tropism during infection. ${ }^{15}$ Therefore, it is suggestive that SP could determine host range of the virus.

Both CQ and HCQ are known to interfere with terminal glycosylation of the Angiotensin Converting Enzyme-2 (ACE-2), a cellular transmembrane receptor (therapeutic target) for the SP on the SARS-CoV-2 expressed on the epithelial surface of the lungs, kidneys, gastrointestinal tract, and blood vessels. It is worthy of note that priming of the SP by the transmembrane protease serine 2 (TMPRSS2) from the virus-target host cell is a crucial step for the fusion between the SP and the ACE-2 on the targeted host cell membrane and entry into the cell. ${ }^{16,17}$
Just like SARS-CoV which is the etiologic agent of Severe Acute Respiratory Syndrome (SARS), SARS-CoV-2 binds with ACE-2 for intracellular invasion. A proteinprotein docking during a computational study revealed that the SP of SARS-CoV-2 has a high binding affinity to the human ACE-2 receptor. ${ }^{18}$ Binding of the virus with this receptor reduces the production of heptapeptide angiotensin (AT1-7) that is known to mediate antiinflammatory, anti-apoptosis, and anti-fibrosis, particularly in the lungs. ${ }^{19}$ Conversely, this interference elevates amounts of angiotensin 2 (AT-2), increases activation of type 1 angiotensin 2 receptor (AT1R), consequent development of acute respiratory syndrome due to the enhanced pro-inflammatory, pro-apoptosis, and pro-fibrosis effects consistent with the activation of AT1R by AT-2. ${ }^{20-22}$ Compounds, including CQ and HCQ that have a significant binding affinity with SP may block the binding of SARS-CoV-2 to ACE-2, which could result in the prevention of the infection. Also, elevation of the $\mathrm{pH}$ at the surface of the effector cell membrane by CQ or HCQ could prevent fusion between the virus and the cell membrane with subsequent prevention of the infection.

A recently conducted in silico study earlier referred to in this review showed that some compounds were able to bind to the ACE- 2 target using virtual screening. Also, the same study revealed that none of the docked compounds could bind with or interrupt the ACE-2-SP complex, suggesting that these compounds might only inhibit ACE2 without interfering with an already on-going infection following the formation of ACE-2-SP complex. ${ }^{13}$ However, these potential ACE-2 inhibitors, including CQ and HCQ may not be suitable for use as drugs in treating SARS-CoV-2 infection based solely on this mechanism of action since this (inhibition of ACE-2) could nullify the protective anti-inflammatory effect of ACE-2 through the AT1-7, a protective factor of lung injury as explained in the preceding statement.

The non-protonated (uncharged) form of CQ or HCQ diffuses freely and quickly across cell membranes into the acidic lysosomes, consequently increasing their $\mathrm{pH} .{ }^{23}$ The accumulation of these drugs in the lysosomes due to protonation and sequestration is referred to as "lysosomotropism". Once the non-protonated drug diffuses into the lysosome, it is protonated (charged), hence unable to diffuse out (ion trapping). This elevates the lysosomal $\mathrm{pH}$ with the resultant inhibition of lysosomal enzymatic functions vital for viral replication. ${ }^{24}$ The consequence is, therefore, the inhibition of nucleic acid replication, glycosylation of viral SP, virus assembly, new virus particle transport, virus release and other processes to achieve its antiviral effects. An in vitro study suggested that the anti-SARS-CoV-2 potency of HCQ is superior to CQ with the former having an EC50 value well below the later. ${ }^{11,25}$ 


\section{Immune-modulatory effects}

The major cause of death from COVID-19 is acute respiratory distress syndrome which is a consistent immune-mediated pathologic event associated with SARS-CoV-2 infection in human. ${ }^{18}$ The SARS-CoV-2induced pneumonia is marked by hyper-activation of effector T cells and excessive production of large amount of pro-inflammatory cytokines, particularly Tumor Necrosis Factor (TNF $\alpha$ ) and interleukins (IL-6, IL-10), a scenario described as "cytokine storm",2,26,27 These facilitate increased vascular permeability and plasma leakage culminating to pulmonary edema, multiple organ failure, and sometimes death. ${ }^{28-30,18}$ The immunemodulatory effect of CQ and HCQ involves suppression of the production and release of these cytokines and to some extent the chemokines that are known to mediate the inflammatory complications of several viral diseases, including COVID-19.

Furthermore, increased lysosomes $\mathrm{pH}$ inhibits immune cell functioning by decreasing chemotaxis, phagocytosis, and superoxide production by the neutrophils. ${ }^{31}$ Sequestration of CQ or HCQ into acidic cellular organelles and elevation of the $\mathrm{pH}$ distorts the normal activities of enzymes, particularly the production of several cytokines, chemokines, or mediators where excessive appearance contributes to the severity of viral infections generally. Thus, by these, CQ or HCQ could be said to have therapeutic potential for treating viral diseases, particularly by ameliorating the associated pathologies like pneumonia caused by the cytokine storm. Again, the suppressing effect observed following treatment of cell culture with CQ pre- or post- exposure to the virus suggests that these agents have prophylactic as well as therapeutic potentials. ${ }^{32,33}$ Consequently, early administration of these proposed-repurposed drugs at clinically relevant doses could avert the progression of COVID-19 pathophysiology to a critical and lifethreatening stage.

\section{ADVERSE EFFECTS OF CHLOROQUINE AND HYDROXYCHLOROQUINE}

Incidence of adverse effects following therapeutic use of CQ and HCQ are rare, nevertheless could be potentially life-threatening. Although the prolonged use of CQ and HCQ in malaria therapy revealed the safety of these drugs in humans, the rare risk of irreversible retinopathy and cardiac arrhythmia cannot be overemphasized. ${ }^{34,35}$ The adverse effects following treatment of patients with CQ or HCQ are due to some of their physicochemical and pharmacokinetic profiles. Ion trapping of these drugs within the lysosomes increasing the lysosomal $\mathrm{pH}$, high lipophilic property, large volume of distribution to tissues (about 65,000 L), high dose-dependent plasma concentrations, long terminal half-life (about 41 days), slow rate of excretion, and long duration of therapy are factors that could contribute to the toxicity associated with $\mathrm{CQ}$ and $\mathrm{HCQ} .{ }^{36} \mathrm{In}$ addition, doses above $2.3 \mathrm{mg} / \mathrm{kg}$ body weight/day for CQ and $5.0 \mathrm{mg} / \mathrm{kg}$ body weight/day for HCQ are reported to be associated with toxicity. ${ }^{37}$

The most serious but uncommon adverse effect of CQ and HCQ is irreversible retinopathy, which depends on the cumulative rather than on the daily dose. ${ }^{38}$ Both CQ and HCQ bind strongly to melanin on the melanin-containing tissues, particularly the retina pigment epithelium and the iris of the eye. The functions of melanin here are absorption of light, prevention of scatter, and protection against free radicals. This melanotrophilic characteristic of these drugs impacts significantly on the susceptibility of patients treated with these drugs to retinopathy. Also, CQor HCQ- related cardiac disorder is rare but is a severe adverse event that could lead to death. However, the adverse effects of CQ and HCQ could be reduced because the coronaviruses are reported to respond to lower doses compared to that used for treatment of malaria. ${ }^{33} \mathrm{~A}$ commonly reported cardiac complication is cardiac arrhythmias. ${ }^{39}$ In all these, the clinical safety profile of HCQ is superior to CQ as well as having lower incidence of drug-drug interactions. ${ }^{37,40}$ This is largely due to its less lipophilicity, smaller volume of distribution, and shorter terminal half-life as compared with CQ.

\section{CONCLUSION}

Chloroquine and hydroxychloroquine could be repurposed for managing COVID-19 cases because they are already extensively used for treating acute non-resistant malaria and auto-immune diseases. Also, a viable vaccine cannot be available in the near future while there is daily rise in morbidity and mortality associated with the disease. Nevertheless, considering the rare but life-threatening concentration-dependent adverse effects of these drugs, and the propensity of emergence of resistant virus strains, it is more appropriate to pause till the final report from several ongoing controlled, randomized, and open-label clinical trials which are aimed at establishing the repurposing claims of these drugs for the treatment of COVID-19. This would circumvent therapeutic errors from the non-judicious use of these drugs and subsequent therapeutic failures as well as adverse effects on patients. This does not discount the prospect of chloroquine and its analogue, hydroxychloroquine bouncing back again to play a prominent role in fighting the pandemic 2019Coronavirus disease which has brought the whole world to a virtual standstill.

\section{Funding: No funding sources \\ Conflict of interest: None declared \\ Ethical approval: Not required}

\section{REFERENCES}

1. WHO (2020): WHO Director-General's opening remarks at the media briefing on COVID 19. https://www.who.int/dg/speeches/details. Accessed on March 28, 2020. 
2. Huang C, Wang Y, Li X. Clinical features of patients infected with 2019 novel coronavirus in Wuhan, China. Lancet. 2020; 395(10223):497-506.

3. Bosak A, Opsenica DM, Šinko G, Zlatar M, Kovarik Z. "Structural aspects of 4-aminoquinolines as reversible inhibitors of human acetylcholinesterase and butyrylcholinesterase". Chemico-Biological Interactions. 2009;308:101-9.

4. Gao J, Tian Z, Yang X. Breakthrough: Chloroquine phosphate has shown apparent efficacy in treatment of COVID-19 associated pneumonia in clinical studies. BioSc Trends. 2020;16:14(1):72-3.

5. Gautret P, Lagier JC, Parola P, Meddeb L, Mailhe M, Doudier B, et al. Hydroxychloroquine and azithromycin as a treatment of COVID-19: results of an open-label non-randomized clinical trial. Int $\mathbf{J}$ Antimicrob Agents. 2020;105949.

6. Wang M, Cao R, Zhang L, Yang X, Liu J, Xu M, et al. Remdesivir and chloroquine effectively inhibit the recently emerged novel coronavirus $(2019-n \mathrm{CoV})$ in vitro. Cell Res 2020.

7. Yao X, Ye F, Zhang M, Cui C, Huang B, Niu P, et al. In vitro antiviral activity and projection of optimized dosing design of hydroxychloroquine for the treatment of severe acute respiratory syndrome coronavirus 2 (SARS-CoV-2). Clin Inf Dis 2020.

8. Ashley EA, Pyae PA, Woodrow CJ. Malaria. The Lancet. 2018;391(10130):1608-621.

9. Rynes RI, Parke AL. Introduction to symposium on antimalarial therapy and lupus. Lupus. 1993;2:S1.

10. Iredale J, Fieger H, Wainer IW. Determination of the stereoisomers of hydroxychloroquine and its major metabolites in plasma and urine following a single oral administration of racemic hydroxychloroquine. Semin Arthritis Rheum. 1993;23:74-81.

11. Savarino A, Boelaert JR, Cassone A, Majori G, Cauda R. Effects of chloroquine on viral infections: an old drug against today's diseases. Lancet Infect Dis. 2003;3:722-27.

12. Wu C, Liu Y, Yang Y, Zhang P, Zhong W, Wang Y et al. Analysis of therapeutic targets for SARS-CoV-2 and discovery of potential drugs by computational methods, Acta Pharmaceutica Sinica B 2020.

13. Bosch BJ, van der Zee R, de Haan CA, Rottier PJ. The coronavirus spike protein is a class I virus fusion protein: structural and functional characterization of the fusion core complex. J Virol. 2003;77:8801-11.

14. Xiaowei L, Manman G, Yizhao P, Liesu M, Shemin L. Molecular immune pathogenesis and diagnosis of COVID-19. J Pharm Anal. Forthcoming 2020.

15. Millet JK, Whittaker GR. Host cell proteases: critical determinants of coronavirus tropism and ophthalmology: Recommendations on screening for chloroquine and hydroxychloroquine. Virus Res. 2015;202:120-34.

16. Matsuyama S, Nagata N, Taguchi F. Efficient activation of the severe acute respiratory syndrome coronavirus spike protein by transmembrane protease TMPRSS2. J Virol. 2020;84(24):12658-64.
17. Matsuyama S, Taguchi F. Two-step conformational change in a coronavirus envelope glycoprotein mediated by receptor binding and proteolysis. J Virol. 2009;83:11133-41.

18. Xu X, Chen P, Wang J, Feng J, Zhou H, Li X, et al. Evolution of the novel coronavirus from the ongoing Wuhan outbreak and modeling of its spike protein for risk of human transmission. Sci China Life Sci. 2020.

19. Ye R, Liu Z. ACE2 exhibits protective effects against LPS-induced acute lung injury in mice by inhibiting the LPS-TLR4 pathway. Exp Mol Pathol. 2019;113:104350.

20. Li W, Moore MJ, Vasilieva N. Angiotensinconverting enzyme 2 is a functional receptor for the SARS coronavirus. Nature. 2013;535-8.

21. Kuba K, Imai Y, Rao S, Jiang C, Penninger JM. A crucial role of angiotensin converting enzyme 2 (ACE2) in SARS coronavirus-induced lung injury. Nat Med. 2005,11:875-879.

22. Kuba K, Imai Y, Rao S, Jiang C, Penninger JM. Lessons from SARS: control of acute lung failure by the SARS receptor ACE2. J Mol Med. 2006;84:81420.

23. Al-Bari MA. Chloroquine analogues in drug discovery: new directions of uses, mechanisms of actions and toxic manifestations from malaria to multifarious diseases. J Antimicrob Chemother 2015;70:1608-21.

24. Kaufmann AM, Krise JP. Lysosomal sequestration of amine-containing drugs: analysis and therapeutic implications. J Pharm Sci. 2007;96:729-46.

25. Schrezenmeier E, Dörner T. Mechanisms of action of hydroxychloroquine and chloroquine: implications for rheumatology. Nat Rev Rheumatol. 2020;16(3):15566.

26. Cameron MJ, Bermejo-Martin, Danesh. Human immunopathogenesis of severe acute respiratory syndrome (SARS). Virus Res. 2008;133:13-9.

27. Channappanavar R, Perlman S. Pathogenic human coronavirus infections: causes and consequences of cytokine storm and immunology. Semin Immunopathol. 2017;39:529-39.

28. Tracey KJ, Cerami A. Tumor necrosis factor: a pleiotropic cytokine and therapeutic target. Annu Rev Med. 1994;45:491-503.

29. Baize S, Leroy EM, Georges AJ, Georges-Courbot MC, Capron M, Bedjabaga I, et al. Inflammatory responses in Ebola virus-infected patients. Clin Exp Immunol. 2002;128:1638.

30. Tanaka T, Narazaki M, Kishimoto T. Immunotherapeutic implications of IL-6 blockade for cytokine storm. Immunotherapy. 2016;8:959-70.

31. Hurst NP, French JK, Gorjatschko L, Betts WH. "Chloroquine and Hydroxychloroquine Inhibit Multiple Sites in Metabolic Pathways Leading to Neutrophil Superoxide Release". J Rheumatol. 1988;15(1):23-7.

32. Keyaerts E, Vijgen L, Maes P, Neyts J, Van Ranst M. In vitro inhibition of severe acute respiratory 
syndrome coronavirus by chloroquine. Biochem Biophys Res Commun. 2004;323:264-8.

33. Vincent MJ, Bergeron E, Benjannet S, Erickson BR, Rollin PE, Ksiazek TG, et al. (2005). Chloroquine is a potent inhibitor of SARS coronavirus infection and spread. Virol J. 2005;2:69.

34. Jaeger A, Sauder I, Kopferschmitt J, Flech K. Clinicalfeatures and management of poisoning due to antimalarial drugs. Med. Toxicol Adverse Drug Exp. 1987;2:242-73.

35. Kalia S, Dutz JP. "New concepts in antimalarial use and mode of action in dermatology". Dermatol Therapy. 2007;20(4):160-74.

36. Paul M, O'PJeill PG, Bray SR, Hawley SA. Aminoquinolines-Past, Present, and Future: A Chemical Perspective. Phmmmol Thu. 1988;77(1):2958.

37. Harrison SW, Konrad P, Barry C. Management of patients undergoing hydroxychloroquine (Plaquenil) therapy. Clin Expt Optometry. 2000;83(1):32-6.
38. Marmor MF, Kellner U, Lai TY, Melles RB, Mieler WF. Recommendations on Screening for Chloroquine and Hydroxychloroquine Retinopathy (2016 Revision). Ophthalmology. 2016;123(6):1386-94.

39. Verny C, De-Gennes C, Sebastien P, Le Thi Houng $\mathrm{Du}$, Chapelon C, Piette JC, et al. Heart conduction disorders in long-term treatment with chloroquine: Two new cases. Presse Med. 1992;21:800-4.

40. Bernstein HN. Ophthalmologic considerations and testing in patients receiving long-term antimalarial therapy. Amer J Med. 1983;75(1):25-34.

Cite this article as: Gberindyer FA, Shima FK, Bosha JA, Ahur VM, Swem FT, Abatan MO.

Repurposing of chloroquine and hydroxychloroquine for the management of COVID-19. Int J Basic Clin Pharmacol 2021;10:300-5. 